Article

\title{
On Invariant Subspaces for the Shift Operator
}

\author{
Junfeng Liu \\ Faculty of Information Technology, Macau University of Science and Technology, Avenida Wai Long, \\ Taipa, Macau 999078, China; jfliu997@sina.com
}

Received: 15 May 2019; Accepted: 28 May 2019; Published: 1 June 2019

\begin{abstract}
In this paper, we improve two known invariant subspace theorems. More specifically, we show that a closed linear subspace $M$ in the Hardy space $H^{p}(\mathbb{D})(1 \leq p<\infty)$ is invariant under the shift operator $M_{z}$ on $H^{p}(D)$ if and only if it is hyperinvariant under $M_{z}$, and that a closed linear subspace $M$ in the Lebesgue space $L^{2}(\partial \mathbb{D})$ is reducing under the shift operator $M_{e^{i \theta}}$ on $L^{2}(\partial \mathbb{D})$ if and only if it is hyperinvariant under $M_{e^{i \theta}}$. At the same time, we show that there are two large classes of invariant subspaces for $M_{e^{i \theta}}$ that are not hyperinvariant subspaces for $M_{e^{i \theta}}$ and are also not reducing subspaces for $M_{e^{i \theta}}$. Moreover, we still show that there is a large class of hyperinvariant subspaces for $M_{z}$ that are not reducing subspaces for $M_{z}$. Furthermore, we gave two new versions of the formula of the reproducing function in the Hardy space $H^{2}(\mathbb{D})$, which are the analogue of the formula of the reproducing function in the Bergman space $A^{2}(\mathbb{D})$. In addition, the conclusions in this paper are interesting now, or later if they are written into the literature of invariant subspaces and function spaces.
\end{abstract}

Keywords: invariant subspace; hyperinvariant subspace; reducing subspace; shift operator; hardy space; lebesgue space; Bergman space

MSC: [2010] 47A15

\section{Introduction and Preliminary}

It is well known that one of the most famous and most intriguing research topics in modern mathematics is the invariant subspace problem. (cf. [1-37] and their references).

In particular, it is worth mentioning that it was the famous computer scientist and mathematician J. von Neumann who initiated the research work of the invariant subspace problem and its applications (cf. [4] and so on).

However, this problem remains still unanswered in Hilbert spaces, but for some concrete operators and classes of operators, there have been affirmative or negative answers. Moreover, the invariant subspace theory has extensive applications in many branches of mathematics as well as other science and technology fields (cf. [1-37] and so on).

Now, we recall some results which are directly and closely related to this paper, and state some key concepts used in this paper.

Let $X$ be a normed space and let $\mathcal{B}(X)$ denote the algebra consisting of all bounded linear operators on $X$. Let $T \in \mathcal{B}(X)$ and let $M$ be a closed linear subspace of $X$. If $T M \subset M$, then $M$ is called an invariant subspace for $T$. In particular, if $M \neq\{0\}$ and $M \neq X$, then $M$ is called a nontrivial invariant subspace for $T$.

Let $T \in \mathcal{B}(X)$ and let $M$ be a closed linear subspace of $X$. If $S M \subset M$ for every $S \in \mathcal{B}(X)$ such that $S T=T S$, then $M$ is called a hyperinvariant subspace for $T$. In particular, if $M \neq\{0\}$ and $M \neq X$, then $M$ is called a nontrivial hyperinvariant subspace for $T$. 
Let $H$ be a Hilbert space and let $T \in \mathcal{B}(H)$. Let $M$ be a closed linear subspace of $H$. If $T M \subset M$ and $T^{*} M \subset M$, then $M$ is called a reducing subspace for $T$, where $T^{*}$ denote the adjoint operator of $T$. In particular, if $M \neq\{0\}$ and $M \neq H$, then $M$ is called a nontrivial reducing subspace.

Let $\mathbb{D}$ and $\partial \mathbb{D}$ denote the open unit disk and the unit circle on the complex plane respectively.

Let $1 \leq p<\infty$. Let $H^{p}(\mathbb{D})$ denote the Hardy space consisting of all analytic functions $f$ such that $\sup _{0<r<1} \int_{0}^{2 \pi}\left|f\left(r e^{i \theta}\right)\right|^{p} d \theta<\infty$ with the norm

$$
\|f\|_{p}=\sup _{0<r<1}\left(\frac{1}{2 \pi} \int_{0}^{2 \pi}\left|f\left(r e^{i \theta}\right)\right|^{p} d \theta\right)^{\frac{1}{p}}=\lim _{r \rightarrow 1^{-}}\left(\frac{1}{2 \pi} \int_{0}^{2 \pi}\left|f\left(r e^{i \theta}\right)\right|^{p} d \theta\right)^{\frac{1}{p}}
$$

and with the inner product

$$
\langle f, g\rangle_{H^{2}(\mathbb{D})}=\lim _{r \rightarrow 1^{-}} \frac{1}{2 \pi} \int_{0}^{2 \pi} f\left(r e^{i \theta}\right) \overline{g\left(r e^{i \theta}\right)} d \theta, \quad f, g \in H^{2}(\mathbb{D}) .
$$

Let $A^{p}(\mathbb{D})$ denote the Bergman space consisting of all analytic functions $f$ such that $\int_{\mathbb{D}}|f(z)|^{p} d A(z)<\infty$ with the norm

$$
\|f\|_{p}(\mathbb{D})=\left(\int_{\mathbb{D}}|f(z)|^{p} d A(z)\right)^{\frac{1}{p}} .
$$

Let $M_{z}$ be the operator on the Hardy space $H^{p}(\mathbb{D})$ defined by

$$
\left(M_{z} f\right)(z)=z f(z), \quad f \in H^{p}(\mathbb{D}) .
$$

Then $M_{z}$ is called the shift operator on the Hardy space $H^{p}(\mathbb{D})$.

If $\phi(z)$ is a function in that Hardy space $H^{\infty}(\mathbb{D})$ such that $\left|\widetilde{\phi}\left(e^{i \theta}\right)\right|=1$ a.e. on $\partial \mathbb{D}$, where $\widetilde{\phi}\left(e^{i \theta}\right)=$ $\lim _{r \rightarrow 1^{-}} \phi\left(r e^{i \theta}\right)$ a.e. on $\partial \mathbb{D}$, then $\phi$ is called an inner function.

In 1949, the pioneering work of A. Beurling's [8] found a description of all invariant subspaces for the shift operator on the Hardy space. It is well known that in the first half of twentieth century, the research interest in Complex Analysis dipped to a low point. It was Beurling's work [8] that revived the research interest of Complex Analysis, and that opened a new research direction of the invariant subspace problem.

We now state the Beurling's theorem.

Theorem A (Beurling's theorem) ([8], [12] p. 246, [18] p. 99, [19,20], [21] p. 82, [24] p. 49, [35] p. 348, [28] p. 42). Let $M_{z}$ be the shift operator on the Hardy space $H^{p}(\mathbb{D})$. Then the following statements are equivalent.

(1). $M$ is a nonzero invariant subspace for $M_{z}$.

(2). There exists an inner function $\phi$ such that $M=\phi H^{p}(\mathbb{D})$.

Moreover, in Theorem A, $\phi$ is uniquely determined by $M$ up to a constant factor of modulus 1.

On the other hand, it is clear that a hyperinvariant subspace for any operator $T$ is a invariant subspace for the operator $T$, but the converse is not necessarily true. For example, let $\phi \in L^{\infty}(\partial \mathbb{D})$ and let $M_{\phi}$ be the multiplication operator on the Lebesgue space $L^{2}(\partial \mathbb{D})$ defined by

$$
\left(M_{\phi} f\right)\left(e^{i \theta}\right)=\phi\left(e^{i \theta}\right) f\left(e^{i \theta}\right), f \in L^{2}(\partial \mathbb{D}) .
$$

Let $e_{n}=e^{i n \theta}$ and $M_{n}=\overline{\operatorname{span}}\left\{e_{n}, e_{n+1}, e_{n+2}, \cdots\right\}, n \in \mathbb{Z}$, where $\mathbb{Z}$ denotes the set of all integers including zero. It is easy to see that every $M_{n}$ is a nontrivial invariant subspace for $M_{e^{i \theta}}$. Moreover, it is clear that $M_{e^{-i \theta}} M_{e^{i \theta}}=I=M_{e^{i \theta}} M_{e^{-i \theta}}$, where $I$ denotes the unit operator on $L^{2}(\partial \mathbb{D})$. Also, since

$$
M_{e^{i \theta}}^{*} M_{n}=M_{e^{-i \theta}} M_{n}=M_{n-1} \supsetneqq M_{n}, n \in \mathbb{Z},
$$


it follows that any $M_{n}$ is not a hyperinvariant subspace for $M_{e^{i \theta}}$ and is also not a reducing subspace for $M_{e^{i \theta}}$.

However, we find every invariant subspace for the shift operator $M_{z}$ on the Hardy space $H^{p}(\mathbb{D})(1 \leq p<\infty)$ is its hyperinvariant subspace (Theorem 1$)$. This result is produced through the interaction of Operator Theory and Complex Analysis, and should have been a basic theory in the invariant subspace problem and Hardy spaces. Since there is no Beurling's theorem in the form of Theorem 1 in the existing literature (cf. $[6,8,11,12,18,21,24,28,35]$, as well as their references and so on), and since Beurling's theorem is one of the most famous theorems in modern mathematics, we shall state this theorem and give an almost self-contained proof in Section 2.

Moreover, there is a reducing subspace theorem in the Lebesgue space $L^{2}(\partial \mathbb{D})$, which is also well known. Let $M_{e^{i \theta}}$ be the operator on $L^{2}(\partial \mathbb{D})$ defined by

$$
\left(M_{e^{i \theta}} f\right)\left(e^{i \theta}\right)=e^{i \theta} f\left(e^{i \theta}\right) \quad f \in L^{2}(\partial \mathbb{D}) .
$$

Then $M_{e^{i \theta}}$ is called the shift operator on $L^{2}(\partial \mathbb{D})$.

Theorem B ([18] p. 111, [21] p. 81, [24] p. 46, [28] p. 39). Let $M_{e^{i \theta}}$ is the shift operator on $L^{2}(\partial \mathbb{D})$. Then the following statements are equivalent.

(1). $M$ is a reducing subspace for $M_{e^{i \theta}}$.

(2). There exists a measurable subset $E$ on $\partial \mathbb{D}$ such that

$$
M=\left\{f \mid f \in L^{2}(\partial \mathbb{D}), f\left(e^{i \theta}\right)=0 \text { a.e. on } E\right\} .
$$

It is easy to see from definitions and basic properties of the reducing subspace and the hyperinvariant subspace that a reducing subspace need not be a hyperinvariant subspace in general.

However, we find every reducing subspace for shift operator $M_{e^{i \theta}}$ on Lebesgue space $L^{2}(\partial \mathbb{D})$ is its hyperinvariant subspace (Theorem 2), which can not be also found in the existing literature (cf. $[6,8,11,18,21,24,28,35]$ as well as their references and so on). In the next section, we will give this result.

Furthermore, we shall show that there is a large classes invariant subspaces for $M_{e^{i \theta}}$ on the Lebesgue space $L^{2}(\partial \mathbb{D})$ that are not hyperinvariant subspaces for $M_{e^{i \theta}}$ and are also not reducing subspaces for $M_{e^{i \theta}}$. At the same time, we still show that there is a large class of hyperinvariant subspaces for $M_{z}$ on the Hardy space $H^{2}(\mathbb{D})$ that are not reducing subspaces for $M_{z}$.

Clearly, the conclusions in this paper are interesting now, or later if they are written into the literature of invariant subspaces and function spaces.

\section{Main Results}

Theorem 1. Let $M_{z}$ be the shift operator on the Hardy space $H^{p}(\mathbb{D}) \quad(1 \leq p<\infty)$. Then the following statements are equivalent.

(1). $M$ is a nonzero invariant subspace for $M_{z}$.

(2). $M$ is a nonzero hyperinvariant subspace for $M_{z}$.

(3). There is an inner function $\phi$ such that $M=\phi H^{p}(\mathbb{D})$.

Moreover, in Theorem 1, $\phi$ is uniquely determined by $M$ up to a constant of modulus 1.

Proof. By definition, every hyperinvariant subspace for $M_{z}$ is its invariant subspace. Thus by Theorem A, to show Theorem 1, it suffice to show that every nonzero invariant subspace $M$ for $M_{z}$ is its hyperinvariant subspace. That is, to show that $A M \subset M$ whenever $A$ ia a bounded linear operator on $H^{p}(\mathbb{D})$ such that $A M_{z}=M_{z} A$. In fact, let $U$ be the operator from $H^{p}(\mathbb{D})$ to $H^{p}(\partial \mathbb{D}),(1 \leq p \leq \infty)$ defined by

$$
(U f)(z)=\widetilde{f}\left(e^{i \theta}\right), \quad f \in H^{p}(\mathbb{D},)
$$


where $\widetilde{f}\left(e^{i \theta}\right)=\lim _{r \rightarrow 1^{-}} f\left(e^{i \theta}\right)$ a.e. on $\partial \mathbb{D}$. It is well known that $U$ is a isometric isomorphism from $H^{p}(\mathbb{D})$ onto $H^{p}(\partial \mathbb{D})$. Moreover, for every bounded linear operator $A$ on $H^{p}(\mathbb{D})$, let

$$
B=U A U^{-1},
$$

then $B$ is a bounded linear operator on $H^{p}(\partial \mathbb{D})$ and is similar to $A$. Let $\left.M_{e^{i \theta}}\right|_{H^{p}(\partial \mathbb{D})}$ is the shift operator on $H^{p}(\partial \mathbb{D})$ defined by

$$
\left(\left.M_{e^{i \theta}}\right|_{H^{p}(\partial \mathbb{D})} f\right)\left(e^{i \theta}\right)=e^{i \theta} f\left(e^{i \theta}\right), \quad f \in H^{p}(\partial \mathbb{D}) .
$$

It is easy to see that $\left.M_{e^{i \theta}}\right|_{H^{p}(\partial \mathbb{D})}=U M_{z} U^{-1}$. Since $A M_{z}=M_{z} A$, it follows that

$$
\begin{aligned}
& \left.B M_{e^{i \theta}}\right|_{H^{p}(\partial \mathbb{D})}=U A U^{-1} U M_{z} U^{-1}=U A M_{z} U^{-1} \\
= & U M_{z} A U^{-1}=\left(U M_{z} U^{-1}\right)\left(U A U^{-1}\right)=\left.M_{e^{i \theta}}\right|_{H^{p}(\partial \mathbb{D})} B
\end{aligned}
$$

That is, the shift operator $\left.M_{e^{i \theta}}\right|_{H^{p}(\partial \mathbb{D})}$ on $H^{p}(\partial \mathbb{D})$ is commutative with the operator $B$. Thus it can be shown by means of a similar method of Theorem 3.2.5 in [24] that there is a function $\widetilde{\psi} \in H^{\infty}(\partial \mathbb{D})$ such that $B=M_{\widetilde{\psi}}$, where $M_{\widetilde{\psi}}$ is the multiplication operator on $H^{p}(\partial \mathbb{D})$ defined by

$$
\left(M_{\widetilde{\psi}} f\right)\left(e^{i \theta}\right)=\widetilde{\psi}\left(e^{i \theta}\right) \widetilde{f}\left(e^{i \theta}\right), \quad \widetilde{f} \in H^{p}(\partial \mathbb{D}) .
$$

Let $\psi=U^{-1} \widetilde{\psi}$, then $\psi \in H^{\infty}(\mathbb{D})$. For each $f \in H^{p}(\mathbb{D})$, let $\widetilde{f}=U f$, then $\widetilde{f} \in H^{p}(\partial \mathbb{D})$ and

$$
\begin{aligned}
& (A f)(z)=\left(U^{-1} B U f\right)(z)=\left(U^{-1} B \widetilde{f}\right)\left(e^{i \theta}\right) \\
= & \left(U^{-1} M_{\widetilde{\psi}} \widetilde{f}\right)\left(e^{i \theta}\right)=\left(U^{-1} \widetilde{\psi} \widetilde{f}\right)\left(e^{i \theta}\right)=\psi(z) f(z)=\left(M_{\psi} f\right)(z) .
\end{aligned}
$$

That is, $A=M_{\psi}$, where $M_{\psi}$ is the multiplication operator on $H^{p}(\mathbb{D})$ defined by

$$
\left(M_{\psi} f\right)(z)=\psi(z) f(z), \quad f \in H^{p}(\mathbb{D}) .
$$

Moreover, it is not difficult to show that if $\psi \in H^{\infty}(\mathbb{D})$ and $f \in H^{p}(\mathbb{D})$, then $\psi f \in H^{p}(\mathbb{D})$. On the other hand, since $M$ is a nonzero invariant subspace, it follows from Theorem A that $M=\phi H^{p}(\mathbb{D})$. This implies that every function $g(z)$ in $M$ has the form $g=\phi f$, where $f \in H^{p}(\mathbb{D})$. Thus we have

$$
A g=A(\phi f)=M_{\psi}(\phi f)=\psi(\phi f)=\phi(\psi f) \in \phi H^{p}(\mathbb{D})=M .
$$

That is $A M \subset M$.

Note 1. It is easy to see from the proof of Theorem 3.2.5 in [24] and Theorem 1 that it is impossible to show that $A=M_{\psi}$ directly in $H^{p}(\mathbb{D})$ by means of the (similar) method of Theorem 3.2 .5 in [24].

Note 2. In 1978, S. Brown [9] has shown that every subnormal operator has a nontrivial invariant subspace, but it has not been known so far whether every subnormal operator has a nontrivial hyperinvariant subspace (cf. [15] p. 169 and so on).

Theorem 2. Let $M_{e^{i \theta}}$ is the shift operator on $L^{2}(\partial \mathbb{D})$. Then the following statements are equivalent.

(1). $M$ is a reducing subspace for $M_{e^{i \theta}}$.

(2). $M$ is a hyperinvariant subspace for $M_{e^{i \theta}}$.

(3). There exists a measurable subset $E$ on $\partial \mathbb{D}$ such that

$$
M=\left\{f \mid f \in L^{2}(\partial \mathbb{D}), f\left(e^{i \theta}\right)=0 \text { a.e. on } E\right\} .
$$


Proof. (a). First of all, we show that $(1) \Rightarrow(2)$. That is, to show that every reducing subspace $M$ for $M_{e^{i \theta}}$ is its hyperinvariant subspace. By definition, it suffice to show that $T M \subset M$ whenever $T$ is a bounded linear operator on $L^{2}(\partial \mathbb{D})$ such that $T M_{e^{i \theta}}=M_{e^{i \theta}} T$. In fact, for each such operator $T$, there is a function $\phi \in L^{\infty}(\partial \mathbb{D})$ such that $T=M_{\phi}$, where $M_{\phi}$ is the multiplication operator on $L^{2}(\partial \mathbb{D})$ defined by (1) ([24] p. 45). Thus it remains to show that $M_{\phi} M \subset M$ for $\phi \in L^{\infty}(\partial \mathbb{D})$.

Indeed, since $M$ is a reducing subspace for $M_{e^{i \theta}}$, it follows from Theorem B that there exists a measurable subset $E$ on $\partial \mathbb{D}$ such that

$$
M=\left\{f \mid f \in L^{2}(\partial \mathbb{D}), f\left(e^{i \theta}\right)=0 \text { a.e. on } E\right\} .
$$

Moreover, for each $f \in M$, let

$$
g\left(e^{i \theta}\right)=\phi\left(e^{i \theta}\right) f\left(e^{i \theta}\right)
$$

Then $g=M_{\phi} f$ and

$$
\begin{aligned}
& \frac{1}{2 \pi} \int_{0}^{2 \pi}\left|g\left(e^{i \theta}\right)\right|^{2} d \theta=\frac{1}{2 \pi} \int_{0}^{2 \pi}\left|\phi\left(e^{i \theta}\right) f\left(e^{i \theta}\right)\right|^{2} d \theta \\
\leq & \|\phi\|_{\infty}^{2} \frac{1}{2 \pi} \int_{0}^{2 \pi}\left|f\left(e^{i \theta}\right)\right|^{2} d \theta=\|\phi\|_{\infty}^{2}\|f\|_{L^{2}(\partial \mathbb{D})}^{2} \\
< & \infty .
\end{aligned}
$$

Also, since $f \in M$, it follows that $f \in L^{2}(\partial \mathbb{D})$ and $f\left(e^{i \theta}\right)=0$ a.e. on $E$. Thus by (2), $g \in L^{2}(\partial \mathbb{D})$ and $g\left(e^{i \theta}\right)=\phi\left(e^{i \theta}\right) f\left(e^{i \theta}\right)=0$ a.e. on $E$. Therefore $M_{\phi} f=g \in M$. Hence $M_{\phi} M \subset M$. This implies that $M$ is a hyperinvariant subspace for $M_{e^{i \theta}}$.

(b). We now show that $(2) \Rightarrow(1)$. In fact, let $M$ be a hyperinvariant subspace for $M_{e^{i \theta}}$. Then $M$ is an invariant subspace for $M_{e^{i \theta}}$. On the other hand, it is easy to see that

$$
M_{e^{i \theta}}^{*} M_{e^{i \theta}}=M_{e^{-i \theta}} M_{e^{i \theta}}=I=M_{e^{i \theta}} M_{e^{-i \theta}}=M_{e^{i \theta}} M_{e^{i \theta}}^{*},
$$

Therefore, $M_{e^{i \theta}}^{*}$ is commutative with $M_{e^{i \theta}}$. Also, since $M$ is a hyperinvariant subspace for $M_{e^{i \theta}}$, $M$ is an invariant subspace for $M_{e^{i \theta}}^{*}$, so that $M$ is a reducing subspace for $M_{e^{i \theta}}$.

(c). By (a), (b) and Theorem B, we obtain Theorem 2.

\section{Some Applications}

As applications of Theorems 1 and 2, we can obtain two corollaries below, which are two nontrivial examples in the invariant subspace problem, and can not be also found in the existing literature (cf. [1-37] as well as their references and so on).

Corollary 1. Let $\phi$ be an inner function, but not a constant function. Then $M=\phi H^{2}(\mathbb{D})$ is a nontrivial hyperinvariant subspace (of course, is a nontrivial invariant subspace) for the shift operator $M_{z}$ on the Hardy space $H^{2}(\mathbb{D})$, but is not a reducing subspace for $M_{z}$.

Proof. Take $\phi_{1}(z) \equiv 1$ for all $z \in \mathbb{D}$. Then $\phi_{1}$ and $\phi$ are two inner functions. We now show that $M \neq H^{2}(\mathbb{D})$. In fact, if $M=H^{2}(\mathbb{D})$, then $\phi H^{2}(\mathbb{D})=H^{2}(\mathbb{D})=\phi_{1} H^{2}(\mathbb{D})$. Thus by the uniqueness in Theorem A, it would follow that $\phi=\phi / \phi_{1}$ is a constant of modulus 1 . This contradicts that $\phi$ is not a constant function, so that $M \neq H^{2}(\mathbb{D})$. Thus by Theorem $1, M=\phi H^{2}(\mathbb{D})$ is a nontrivial hyperinvariant subspace for $M_{z}$. Also since the only reducing subspace for $M_{z}$ are $\{0\}$ and the whole space $H^{2}(\mathbb{D})\left([28]\right.$ p. 38, Theorem 3.5), $M$ is not a reducing subspace for $M_{z}$.

Corollary 2. Let $\phi$ be a function in the lebesgue space $L^{\infty}(\partial \mathbb{D})$ such that $\left|\phi\left(e^{i \theta}\right)\right|=1$ a.e. on $\partial \mathbb{D}$. Then $M=\phi H^{2}(\partial \mathbb{D})$ is a nontrivial invariant subspace for the shift operator $M_{e^{i \theta}}$ on the Lebesgue space $L^{2}(\partial \mathbb{D})$, but is not a hyperinvariant subspace for $M_{e^{i \theta}}$ and is also not a reducing subspace for $M_{e^{i \theta}}$. 
Proof. It is well known that $M$ is a nonreducing invariant subspace for $M_{e^{i \theta}}$ if and only if there exists a function $\phi \in L^{\infty}(\partial \mathbb{D})$ with $\left|\phi\left(e^{i \theta}\right)\right|=1$ a.e. on $\partial \mathbb{D}$ such that $M=\phi H^{2}(\mathbb{D})$ (see [28] p. 40, Theorem 3.9). Thus by Theorem 2, the conclusion is obtained.

\section{Two Formulas of the Reproducing Function}

It is well known that there are two formulas of the reproducing function in the Hardy space $H^{2}(\mathbb{D})$. More specifically, if $f(z)$ is a function in $H^{2}(\mathbb{D})$, then we have

(a). the Cauchy integral formula

$$
f(z)=\frac{1}{2 \pi} \int_{0}^{2 \pi} \frac{\widetilde{f}\left(e^{i \theta}\right)}{1-z e^{-i \theta}} d \theta, z \in \mathbb{D}
$$

(b). the Poisson integral formula

$$
\begin{aligned}
f(z) & =\frac{1}{2 \pi} \int_{0}^{2 \pi} \frac{1-|z|^{2}}{\left|1-z e^{-i \theta}\right|^{2}} \widetilde{f}\left(e^{i \theta}\right) d \theta \\
& =\frac{1}{2 \pi} \int_{0}^{2 \pi} \frac{1-\rho^{2}}{1-2 \rho \cos (t-\theta)+\rho^{2}} \widetilde{f}\left(e^{i \theta}\right) d \theta, \quad z=\rho e^{i t} \in \mathbb{D} .
\end{aligned}
$$

where $\widetilde{f}\left(e^{i \theta}\right)=\lim _{r \rightarrow 1^{-}} f\left(r e^{i \theta}\right)$ a.e. on $\partial \mathbb{D}$. It is also well known that there is a formula of the reproducing function in the Bergman space $A^{2}(\mathbb{D})$. More precisely, if $f(z)$ is a function in $A^{2}(\mathbb{D})$, then we have the Bergman integral formula

$$
f(z)=\frac{1}{\pi} \int_{\mathbb{D}} \frac{f(\omega)}{(1-z \bar{\omega})^{2}} d A(\omega), \quad z \in \mathbb{D} .
$$

Note that in the formula (5), the reproducing function $f(z)$ is obtained by means of the integral of the function value of $f$ in $\mathbb{D}$; while in the formulas (3) and (4), the reproducing function $f(z)$ is obtained by means of the integral of the boundary function $\widetilde{f}\left(e^{i \theta}\right)$ of $f$. But we find that the reproducing function $f(z)$ in the Hardy space can be also obtained by means of the integral of the function value of $f$ in $\mathbb{D}$ as in the Bergman space. More specifically, if $f(z)$ is a function in $H^{2}(\mathbb{D})$, then we have the following two formulas of the reproducing function.

$\left(a^{\prime}\right)$

$$
f(z)=\lim _{r \rightarrow 1^{-}} \frac{1}{2 \pi} \int_{0}^{2 \pi} \frac{f\left(r e^{i \theta}\right)}{1-z r e^{-i \theta}} d \theta, z \in \mathbb{D} ;
$$

$\left(b^{\prime}\right)$

$$
\begin{aligned}
f(z) & =\lim _{r \rightarrow 1^{-}} \frac{1}{2 \pi} \int_{0}^{2 \pi} \frac{1-|z|^{2}}{\left|1-z r e^{-i \theta}\right|^{2}} f\left(r e^{i \theta}\right) d \theta \\
& =\lim _{r \rightarrow 1^{-}} \frac{1}{2 \pi} \int_{0}^{2 \pi} \frac{1-\rho^{2}}{1-2 \rho r \cos (t-\theta)+\rho^{2} r^{2}} f\left(r e^{i \theta}\right) d \theta, \quad z=\rho e^{i t} \in \mathbb{D} .
\end{aligned}
$$

Since there is no mention of the formulas (6) and (7) in the existing literature (cf. [11,18-21,24,35] as well as their references and so on), we write down and prove these two formulas here, for sake of comparison with the formula of the reproducing function in the Bergman space, and for convenience of later references.

Now we give the proof of formulas (6) and (7).

Proof. For each $z \in \mathbb{D}$, write

$$
K_{z}(\omega)=\frac{1}{1-\bar{z} \omega}, \quad \omega \in \mathbb{D},
$$




$$
P_{z}(\omega)=\frac{1-|z|^{2}}{|1-\bar{z} \omega|^{2}}, \quad \omega \in \mathbb{D} .
$$

Then $K_{z} \in H^{2}(\mathbb{D}), P_{z} \in H^{2}(\mathbb{D})$. Therefore there exist the radial limits

$$
\widetilde{K}_{z}\left(e^{i \theta}\right)=\lim _{r \rightarrow 1^{-}} K_{z}\left(r e^{i \theta}\right)=\frac{1}{1-\bar{z} e^{i \theta}} \text { a.e. on } \partial \mathbb{D}
$$

and

$$
\widetilde{P}_{z}\left(e^{i \theta}\right)=\lim _{r \rightarrow 1^{-}} P_{z}\left(r e^{i \theta}\right)=\frac{1-|z|^{2}}{\left|1-\bar{z} e^{i \theta}\right|^{2}} \text { a.e. on } \partial \mathbb{D},
$$

and $\widetilde{K_{z}} \in H^{2}(\partial \mathbb{D}) \subset L^{2}(\partial \mathbb{D}), \widetilde{P}_{z} \in H^{2}(\partial \mathbb{D}) \subset L^{2}(\partial \mathbb{D})$. Thus by formulas (3) and (4) as well as the properties of the Hardy space, we have

$$
\begin{aligned}
f(z) & =\frac{1}{2 \pi} \int_{0}^{2 \pi} \frac{\widetilde{f}\left(e^{i \theta}\right)}{1-z e^{-i \theta}} d \theta=\left\langle\widetilde{f}, \widetilde{K_{z}}\right\rangle_{L^{2}(\partial \mathbb{D})} \\
& =\left\langle f, K_{z}\right\rangle_{H^{2}(\mathbb{D})}=\lim _{r \rightarrow 1^{-}} \frac{1}{2 \pi} \int_{0}^{2 \pi} \frac{f\left(r e^{i \theta}\right)}{1-z r e^{-i \theta}} d \theta, \quad z \in \mathbb{D}
\end{aligned}
$$

and

$$
\begin{aligned}
f(z) & =\frac{1}{2 \pi} \int_{0}^{2 \pi} \frac{1-|z|^{2}}{\left|1-z e^{-i \theta}\right|^{2}} \widetilde{f}\left(e^{i \theta}\right) d \theta \\
& =\left\langle\widetilde{f}, \widetilde{P}_{z}\right\rangle_{L^{2}(\partial \mathbb{D})}=\left\langle f, P_{z}\right\rangle_{H^{2}(\mathbb{D})} \\
& =\lim _{r \rightarrow 1^{-}} \frac{1}{2 \pi} \int_{0}^{2 \pi} \frac{1-|z|^{2}}{\left|1-z r e^{-i \theta}\right|^{2}} f\left(r e^{i \theta}\right) d \theta \\
& =\lim _{r \rightarrow 1^{-}} \frac{1}{2 \pi} \int_{0}^{2 \pi} \frac{1-\rho^{2}}{1-2 \rho r \cos (t-\theta)+\rho^{2} r^{2}} f\left(r e^{i \theta}\right) d \theta, \quad z=\rho e^{i \theta} \in \mathbb{D},
\end{aligned}
$$

where $\langle\cdot, \cdot\rangle_{L^{2}(\partial \mathbb{D})}$ denote the inner product in the Lebesgue space $L^{2}(\partial \mathbb{D})$.

\section{Conclusions}

The main conclusions of this paper are as follows:

(1). $M$ is an invariant subspace for the shift operator $M_{z}$ on $H^{p}(\mathbb{D})(1 \leq p<\infty)$ if and only if it is a hyperinvariant subspace for $M_{z}$.

(2). $\quad M$ is a reducing subspace for the shift operator $M_{e^{i \theta}}$ on $L^{2}(\partial \mathbb{D})$ if and only if it is a hyperinvariant subspace for $M_{e} i \theta$.

(3). Every function $f$ in $H^{p}(\mathbb{D}) \quad(1 \leq p<\infty)$ can be reproduced by the integral of the function value of $f$ in $\mathbb{D}$ as in the Bergman space.

Funding: This project was partially supported by the Macao Science and Technology Development Fund (No.186/2017/A3).

Conflicts of Interest: The author declares no conflicts of interest.

\section{References}

1. Abramovich, Y.A.; Aliprantis, C.D. An Invitation to Operator Theory; American Mathematical Socety: Providence, RI, USA, 2002.

2. Ambrozie, C.; Müller, V.M. Invariant subspaces for polynomially bounded operators. J. Funct. Anal. 2004, 213, 321-345. [CrossRef]

3. Argyros, S.A.; Haydon, R.G. A hereditarily indecomposable $\mathcal{L}_{\infty}$-space that solves the scalar-plus-compact problem. Acta Math. 2011, 206, 1-54. [CrossRef] 
4. Aronszajn, N.; Smith, K.T. Invariant subspaces of completely continuous operators. Ann. Math. 1954, 60, 345-350. [CrossRef]

5. Ball, J.A.; Bolotnikov, V. Weighted Bergman spaces: Shift-invariant subspaces and input/state/output linear systems. Integr. Equ. Oper. Theory 2013, 76, 301-356. [CrossRef]

6. Beauzamy, B. Introduction to Operator Theory and Invariant Subspaces; North-Holland: New York, NY, USA, 1988.

7. Bernstein, A.R.; Robinson, A. Solution of an invariant subspace problem of K. T. Smith and P. R. Halmos. Pac. J. Math. 1966, 16, 421-431. [CrossRef]

8. Beurling, A. On two problems concerning linear transformations in a Hilbert space. Acta Math. 1949, 81, 239-255. [CrossRef]

9. Brown, S. Some invariant subspaces for subnormal operators. Integr. Equ. Oper. Theory 1978, 1, $310-333$. [CrossRef]

10. Brown, S. Hyponormal operators with thick spectra have invariant subspaces. Ann. Math. 1987, 125, $93-103$. [CrossRef]

11. Duren, P.L. Theorey of $H^{p}$ Spacce; Academic Press: New York, NY, USA, 1970; Dover Publications Inc.: New York, NY, USA, 2000.

12. Duren P.L.; Schuster, A. Bergman Spaces, Math. Surveys Monographs; American Mathematical Socety: Providence, RI, USA, 2004.

13. Enflo, P. On the invariant subspace problem for Banach spaces. Acta Math. 1987, 158, 213-313. [CrossRef]

14. Eschmeier, J.; Prunaru, B. Invariant subspaces for operators with Bishop's property $(\beta)$ and thick spectrum. J. Funt. Anal. 1990, 94, 196-222. [CrossRef]

15. Eschmeier, J. Introduction to Banach Algebras, Operators, and Harmonic Analysis-Invariant Spaces; Cambridge University Press: Cambridge, UK, 2003.

16. Halmos, P.R. Invariant subspaces of polynomially compact operators. Pac. J. Math. 1966, 16, $433-437$. [CrossRef]

17. Halmos, P.R. A Hilbert Space Problem Book, 2nd ed.; Springer-Verlag: New York, NY, USA, 1982.

18. Hoffman, K. Banach Spaces of Analytic Functions; Prentice Hall Inc.: Englewood Cliffs, NJ, USA, 1962; Dover Publications Inc.: New York, NY, USA, 1988.

19. Karaev, M.T. On the proof of Beurling's theorem on z-invariant subspaces. Expos. Math. 2007, 25, $265-267$. [CrossRef]

20. Karaev, M.T. On a Beurling-Arveson type theorem for some functional Hilbert spaces and related questions. Integr. Equ. Oper. Theory 2008, 62, 77-84. [CrossRef]

21. Koosis, P. Introduction to $H^{p}$ spaces; Cambridge University Press: Cambridge, UK, 1998.

22. Liu, J. On invariant subspaces for polynomially bounded operators. Czechoslov. Math. J. 2017, 67, 1-9. [CrossRef]

23. Lomonosov, V. Invariant subspaces of the family of operators that commute with a completely continuous operator. Funct. Anal. Appl. 1973, 7, 213-214. [CrossRef]

24. Martínez-Avendaño, R.A.; Rosenthal, P. An Introduction to Operators on the Hardy-Hilbert Space; Springer-Verlag: New York, NY, USA, 2007.

25. Michaels, A.J. Hilden's simple proof of Lomonosov's invariant subspace theorem. Adv. Math. 1977, 25, 56-58. [CrossRef]

26. Popov, A.I.; Tcaciuc, A. Every operator has almost-invariant subspaces. J. Funct. Anal. 2013, 265, $257-265$. [CrossRef]

27. Radjavi, H.; Rosenthal, P. On invariant subspaces and reflexive algebras. Am. J. Math. 1969, 91, 683-692. [CrossRef]

28. Radjavi, H.; Rosenthal, P. Invariant Subspaces, 2nd ed.; Dover Publications Inc.: New York, NY, USA, 2003.

29. Radjavi, H.; Troitsky, V.G. Invariant sublattices. Ill. J. Math. 2008, 52, 437-462. [CrossRef]

30. Read, C.J. A solution to the invariant subspace problem. Bull. Lond. Math. Soc. 1984, 16, 337-401. [CrossRef]

31. Read, C.J. A solution to the invariant subspace problem on the space $l^{1}$. Bull. Lond. Math. Soc. 1985, 17, 305-317. [CrossRef]

32. Read, C.J. A short proof concerning the invariant subspace problem. J. Lond. Math. Soc. 1986, 34, $335-348$. [CrossRef]

33. Read, C.J. Quasinilpotent operators and the invariant subspace problem. J. Lond. Math. Soc. 1997, 56, 595-606. [CrossRef] 
34. Rezaei, H. On operators with orbits dense relative to nontrivial subspaces. Funct. Anal. Its Appl. 2017, 51, 112-122. [CrossRef]

35. Rudin, W. Real and Complex Analysis, 3rd ed.; McGraw-Hill Companies, Inc.: Columbus, OH, USA, 1987.

36. Shimorin, S.M. Wold-type decompositions and wandering subspaces for operators close to isometries. J. Reine Angew. Math. 2001, 531, 147-189. [CrossRef]

37. Shimorin, S.M. On Beurling-type theorems in weighted $l^{2}$ and Bergman Spaces. Proc. Am. Math. Soc. 2002, 131, 1777-1787. [CrossRef]

(C) 2019 by the authors. Licensee MDPI, Basel, Switzerland. This article is an open access article distributed under the terms and conditions of the Creative Commons Attribution (CC BY) license (http:/ / creativecommons.org/licenses/by/4.0/). 